Linguistique, littérature, didactique

\title{
Mésusages du don et interventions du surnaturel dans quelques textes des $\mathrm{XII}^{\mathrm{e}}$ et $\mathrm{XIII}^{\mathrm{e}}$ siècles
}

\section{Sophie Albert}

\section{OpenEdition Journals}

Édition électronique

URL : http://journals.openedition.org/pratiques/1812

DOI : 10.4000/pratiques. 1812

ISSN : 2425-2042

\section{Éditeur}

Centre de recherche sur les médiations (CREM)

\section{Édition imprimée}

Date de publication : 15 décembre 2011

Pagination : 117-128

\section{Référence électronique}

Sophie Albert, " Mésusages du don et interventions du surnaturel dans quelques textes des $\mathrm{II}^{\mathrm{e}} \mathrm{et} \mathrm{XII \| ^{ \textrm {e } }}$ siècles », Pratiques [En ligne], 151-152 | 2011, mis en ligne le 16 juin 2014, consulté le 01 mai 2019. URL : http://journals.openedition.org/pratiques/1812; DOI : 10.4000/pratiques.1812 


\title{
Mésusages du don et interventions du surnaturel dans quelques textes des $\mathrm{XII}^{\mathrm{e}}$ et $\mathrm{XIII}^{\mathrm{e}}$ siècles ${ }^{(1)}$
}

\section{Sophie Albert}

\author{
Université de Paris IV-Sorbonne - EA 4349
}

Cet article est issu d'une expérience pédagogique engagée en 2008-2009 à ParisDiderot et poursuivie en 2009-2010 à l'Université d'Angers, auprès d'étudiants de deuxième année de licence. Le cours visait à initier les étudiants à la littérature des XII ${ }^{\mathrm{e}}$ $\mathrm{XIII}^{\mathrm{e}}$ siècles et aux phénomènes de réécriture qui la caractérisent. Plus précisément, il visait à étudier la manière dont chaque genre ou chaque œuvre singulière reprend et réagence des thèmes ou des motifs récurrents dans les textes médiévaux, pour en faire varier la signification. À cet effet, il était organisé selon des entrées choisies d'une part pour leur fonction narrative au sein des œuvres, d'autre part pour leur faculté à cristalliser des tensions ou des déterminations idéologiques sous-jacentes. Parmi ces entrées figurait la question des échanges et du don, qui occupe une place essentielle dans la société médiévale et dans les récits qu'elle a produits. Cette question sera ici appréhendée à travers quatre textes des XII ${ }^{\mathrm{e}}-\mathrm{XIII}{ }^{\mathrm{e}}$ siècles : le lai de Lanval attribué à Marie de France, centré sur le pacte amoureux entre le chevalier Lanval et une femme-fée (ca. 1170); la vie de Gerbert rapportée par Gautier Map dans le De nugis curialium, selon laquelle Gerbert, après avoir conclu un pacte avec une femme diabolique, s'enrichit et gravit les échelons de la cléricature jusqu'à devenir pape sous le nom de Silvestre II (fin du XII ${ }^{\mathrm{e}}$ siècle); le Miracle de Théophile composé par Rutebeuf, où le clerc Théophile regagne fortune et honneurs en se soumettant au démon (ca. 1260); les versions latine et française de la légende du Volto Santo de Lucques, dans lesquelles l'effigie du Christ rétribue un jongleur en lui lançant son soulier d'or (XIII ${ }^{\mathrm{e}}$ siècle $)^{(2)}$.

(1) Je tiens à remercier très chaleureusement Jean-Pierre Albert, Marlène Albert-Llorca et Patrick Henriet, qui ont bien voulu relire cet article et me faire part de leurs remarques. Je remercie également Gisèle Besson, qui a revu et corrigé les traductions des textes latins cités dans le texte ou en note.

(2) Je m'appuie sur les éditions suivantes : Marie de France, Lais, éd. Karl Warnke, trad. Laurence HarfLancner, Paris, Librairie Générale Française, 1990, pp. 134-167 ; Walter Map, De nugis curialium, éd. Montaque R. James, révisé par Christopher N. L. Brooke et Roger A. B. Mynors, Oxford, Clarendon Press, 1983, trad. française d'Alan Keith Bate, Gautier Map, Contes pour les gens de cour, Turnhout, Brepols, 1993 ; Rutebeuf, Le Miracle de Théophile, éd. et trad. Jean Dufournet, Paris, Flammarion, 1987 ; Wendelin Foerster, «Le saint Vou de Luques », Mélanges Chabaneau, Genève, Slatkine Reprints, 1973 [impr. originale : 1907], pp. 1-56, pp. 32-52 pour l'édition partielle du récit français, pp. 52-54 pour le récit latin. 
Ces textes proviennent de milieux divers, et ressortissent à différents domaines linguistiques et génériques : il peut paraître a priori étrange de les réunir en une même étude. Cette réunion est justifiée par deux points de convergence, étroitement articulés : chacun conjoint, suivant des modalités spécifiques, un mésusage du don et l'intervention d'un être surnaturel. Au-delà de ce point commun, leur comparaison mettra au jour des déplacements dont il faudra comprendre le sens et la portée. Je procéderai pour ce faire selon les principes de l'analyse sérielle, théorisés par Jérôme Baschet pour l'étude de l'image médiévale : le motif du don sera envisagé à travers plusieurs actualisations constituées en série, dont je dégagerai les constantes et les variations ${ }^{(3)}$. Outre cette méthode, élaborée à partir de l'analyse structurale de Claude Lévi-Strauss ${ }^{(4)}$, j'emprunterai encore de deux manières au champ de l'anthropologie sociale. À un premier niveau, j'aurai recours aux outils conceptuels définis par Marcel Mauss dans l'Essai sur le $d o n^{(5)}$. À un second niveau, je m'appuierai sur les transpositions de ces outils par les historiens des représentations, qui prennent en compte le contexte dans lequel les œuvres sont produites et reçues ; cette prise en compte implique d'adapter les paradigmes interprétatifs forgés par les anthropologues et, parfois, de les abandonner pour en inventer d'autres. De la même manière, les textes retenus me conduiront à mettre à l'épreuve la théorie de Marcel Mauss, puis à y surimposer des interprétants propres au Moyen Âge chrétien. Par souci de clarté, j' analyserai successivement chacun de ces textes en une progression menant du plus simple, le lai de Lanval, aux histoires autrement complexes du Volto Santo.

\section{Recourir au paradigme : le chevalier et la fée}

Dans les sociétés étudiées par Marcel Mauss, le don, bien qu'il se présente sous l'apparence trompeuse de la gratuité et de la générosité, est en réalité soumis à une pression sociale qui l'érige en obligation : l'enjeu, pour celui qui donne, est de ne pas déroger à son rang et de se montrer plus généreux que ses pairs. Le don, ainsi, mesure et prouve tout à la fois le pouvoir du donateur, le plus offrant étant aussi le plus puissant. Ce mécanisme, indissociable d'une société de face-à-face, s'applique assez heureusement à la valorisation féodale de la largesse. La théorie de Mauss constitue notamment un cadre d'interprétation pertinent pour le lai de Lanval, qui promeut des valeurs courtoises et aristocratiques. Le roi Arthur, représenté au début du récit distribuant à ses barons des terres et des femmes, obéit à une obligation de donner dont Philippe Haugeard a relevé d'autres exemples épiques et romanesques ${ }^{(6)}$. En oubliant de rétribuer Lanval, il interrompt la circulation des richesses et manque à son devoir de largesse à l'encontre du chevalier. Comme l'a noté Carlos F. Clamote Carreto, ce scénario doit être replacé dans un groupe de récits qui dénonce « les effets pernicieux du don refusé ou perverti, ainsi que ses implications sur le plan politique, social et poétique ${ }^{(7)}$. Dans cette perspective, la défaillance du roi est « surcompensée par le merveilleux féerique qui permettra

(3) Jérôme Baschet, «Inventivité et sérialité des images médiévales. Pour une approche iconographique élargie », Annales HSS 51, 1996, pp. 93-133.

(4) Voir notamment « La structure et la forme », Anthropologie structurale deux, Paris, Plon, 1973, pp. 139-173.

(5) Essai réédité dans Marcel Mauss, Sociologie et anthropologie, Paris, Presses Universitaires de France, 2004 [première éd. : 1929 ; aux PUF : 1950], pp. 142-279.

(6) Philippe Haugeard, «L'enchantement du don. Une approche anthropologique de la largesse royale dans la littérature médiévale (XII ${ }^{\mathrm{e}}$-XIII ${ }^{\mathrm{e}}$ siècles) », Cahiers de Civilisation Médiévale 195, 2006, pp. 295-312.

(7) Carlos Fonseca Clamote Carreto, « Le don en anamorphose ou la réécriture du monde. Configurations et enjeux de 1'hospitalité dans le récit médiéval (XII - XIII ${ }^{\mathrm{e}}$ siècles) », COnTEXTES [En ligne], $\mathrm{n}^{\circ} 5$, mai 2009, mis en ligne le 25 mai 2009, URL : http://contextes.revues.org/index4250.html, § 4 . 
au héros de recréer, dans toute sa plénitude, l' "atmosphère du don", selon la terminologie de M. Mauss, et, par ce biais, de colmater la rupture symbolique et sociale ${ }^{(8)}$.

De fait, la fée dont Lanval devient bientôt l'amant, en accordant au chevalier l'aisance matérielle, pallie la déficience du roi. Son intervention ne peut cependant être comprise comme un pur élan de générosité. Elle recèle bien au contraire un rapport de domination qui transparaît de deux manières. D'une part, le don de la richesse ne va pas sans un contre-don : la fée exige de Lanval qu'il lui déclare allégeance, ce qui le place explicitement dans une position de soumission analogue à celle d'un vassal vis-à-vis de son suzerain. D'autre part, ce don est étroitement subordonné à un interdit caractéristique des récits féeriques, en vertu duquel le chevalier ne doit révéler à personne l'existence de la fée. La transgression de cet interdit par Lanval ne rend que plus manifeste l'ascendant de sa maîtresse : l'existence du chevalier, d'abord menacée par la disparition soudaine de la fée, est pour finir sauvée par son retour. Aussi la domination inhérente au don surnaturel n'est-elle nullement "enchantée ", suivant l'acception que Pierre Bourdieu confère à ce terme ${ }^{(9)}$; sa violence ressort du pouvoir de vie et de mort que la fée exerce sur Lanval.

Cette domination offre néanmoins à Lanval de précieuses contreparties. Grâce à la fée, le héros recouvre la faculté de donner largement, sans pour autant s'exposer à la crainte de la pénurie. En effet, comme chez Marcel Mauss, « cum plus despendra richement, / e plus avra or e argent» (v. 141-142) ${ }^{(10)}$ : par une sorte de vertu magique, la prodigalité rapporte, au sens financier du terme. À partir de ce moment-là, les richesses circulent selon un double mouvement : le don va de la fée à Lanval, puis de Lanval au reste du monde. Outre de l'or et de l'argent, la fée procure au héros des habits (v. 174$175,202)$ et le sert magnifiquement (v. 183, 192). Ces bienfaits sont ensuite redirigés vers l'extérieur par Lanval :
N'ot en la vile chevalier
ki de surjur ait grant mestier,
que il ne face a lui venir
e richement e bien servir.
Lanval donout les riches duns,
Lanval aquitout les prisuns,
Lanval vesteit les jugleürs,
Lanval faiseit les granz honurs,
Lanval despendeit largement,
Lanval donout or e argent ;
n'i ot estrange ne privé
a qui Lanval n'eüst doné ${ }^{(11)}$. (v. 205-216)

Carlos F. Clamote Carreto rapproche cette anaphore d'un développement similaire par lequel le Roman de Brut décrit la largesse d'Arthur ${ }^{(12)}$. Si la proximité des deux passages n'autorise pas à conclure à une réécriture consciente et concertée du roman par le lai, elle permet du moins de souligner un glissement, de la figure du roi vers celle du chevalier. Ce glissement est confirmé par un autre indice. Les largesses de Lanval coïn-

(8) Ibid., n. 14.

(9) Voir notamment Le Sens pratique, Paris, Les Éditions de Minuit, 1980, p. 167-207.

(10) «Plus il se répandra en largesses, plus il aura d'or et d'argent !» (trad. cit., p. 141).

(11) «Dans la ville, il n'est chevalier dans le besoin qu'il ne fasse venir chez lui pour mettre sa richesse à son service. Lanval distribue de riches dons, Lanval paie les rançons des prisonniers, Lanval habille les jongleurs, Lanval prodigue les honneurs, Lanval multiplie les largesses, Lanval donne or et argent ; étrangers ou gens du pays, tous ont reçu un don de lui » (trad. cit., p. 145).

(12) «Le don en anamorphose...», art. cit., n. 14. 
cident partiellement avec le septénaire des œuvres de miséricorde, dont la liste se fixe au cours du XII ${ }^{\mathrm{e}}$ siècle. Selon ce septénaire, le gouvernant idéal doit visiter, abreuver, nourrir, racheter, vêtir, panser et ensevelir ${ }^{(13)}$. Lanval, de son côté, nourrit et, peut-on supposer, abreuve les étrangers ; il rachète les prisonniers et habille les jongleurs. Par rapport aux prescriptions des clercs, et hormis le silence touchant aux autres œuvres de miséricorde, le lai introduit un déplacement : tandis que le septénaire est habituellement employé pour codifier les devoirs du prince, il concerne ici un simple chevalier. Ce déplacement est révélateur de la position nouvellement acquise par le héros, qui remplace et surpasse Arthur dans sa fonction de dispensateur de biens.

La rivalité des deux personnages s'exprime également à travers un jeu d'échos et de variantes entre la Pentecôte initiale, fête du don par excellence ${ }^{(14)}$, et l'anaphore ci-dessus. Si Arthur, dans la scène d'ouverture, séjourne à Cardueil (v. 5), Lanval, en un geste plus altruiste, fournit le « surjur» aux chevaliers dans le besoin ${ }^{(15)}$. Si le roi « asez i duna riches duns » (v. 13), Lanval, de son côté, « donout les riches duns », l'imparfait suggérant que ces dons ne sont pas circonscrits à une occasion ponctuelle. Enfin, là où Arthur s'avère incapable de traiter dignement cet « huem estranges » (v. 36) qu' est Lanval, le héros, faisant mieux que le roi, accueille y compris les « estrange». Le don tient ainsi un rôle de premier plan dans les rapports de force entre le roi et le chevalier. Encore accentuée par la concurrence entre la beauté de la reine et celle de la fée, la tension agonistique entre Arthur et Lanval se résout au profit du second : pourvu d'une évidente visée compensatoire, le lai met en scène le pouvoir fantasmé de la classe chevaleresque.

\section{Adapter le paradigme : le clerc et le démon}

Si l'on peut analyser Lanval à la lumière des principes mis au jour par Marcel Mauss, c'est d'abord parce que la société féodale partage avec les sociétés étudiées par cet anthropologue une même culture de l'honneur ; c'est aussi parce que les biens matériels, objets des échanges et du don, y sont pourvus d'une valeur reconnue par l'ensemble des acteurs engagés dans la transaction. Dès lors que ce présupposé cesse d'être effectif, c'est-à-dire dès lors que la valeur des biens matériels est sujette à caution, les fondements mêmes de la théorie maussienne s'en trouvent ébranlés. Or le christianisme, en établissant un rapport hiérarchique entre la cité terrestre et la cité céleste, conduit à relativiser et, parfois, à nier la valeur du monde matériel : légitime et même souhaitable quand elle concerne les richesses de l'Église, la possession des biens terrestres est en revanche critiquable quand elle concerne l'individu, dont elle compromet le salut spirituel ${ }^{(16)}$. Cet élément infléchit profondément le sens des récits présentant conjointement un mésusage du don et un échange avec un être surnaturel.

L'histoire de Gerbert, telle que la rapporte Gautier Map dans le De nugis curialium, offre une illustration éloquente à cet infléchissement. Gerbert, un écolâtre, s'éprit de la fille du prévôt, qui repoussa ses avances. Aveuglé par son amour néfaste, le héros négligea ses affaires, au point de tomber dans une extrême misère matérielle et morale : acca-

(13) Sur la place des œuvres du septénaire dans les miroirs des princes et les actions royales, voir Priscille Aladjidi, Le roi père des pauvres, France $X I I I^{e}-X V^{e}$ siècles, Rennes, Presses Universitaires de Rennes, 2009.

(14) L'ouverture célèbre du Chevalier au Lion de Chrétien de Troyes, qui décrit la "Penthecouste » comme «chele feste qui tant couste» (v. 5-6), correspond à une réalité de la société féodale : la Pentecôte était l'occasion pour les seigneurs d'adouber des chevaliers et de faire montre de largesse.

(15) $C f$. aussi, du côté d'Arthur, le v. 12 (« i aveit li reis surjurné », où le roi est encore sujet du verbe) et, du côté de Lanval, le v. 203 (le héros tient «bon ostel»).

(16) Sur la légitimation des richesses de l'Eglise et son articulation avec la critique de l'enrichissement personnel, voir Patrick Henriet, «En quoi peut-on parler d'une spiritualité de la Réforme grégorienne ? », Revue d'Histoire de l'Église de France, t. 96 (n²36), janvier-juin 2010, pp. 71-91. 
blé de dettes, abandonné de tous, il « se trouva sans un sou chez lui, où, hirsute et sale, ni rasé ni coiffé, il négligea sa personne » ${ }^{(17)}$. Un jour, quittant la ville à midi, il partit se promener dans la forêt. Il y rencontra « une femme d'une beauté inouïe, assise sur un grand tapis de soie, avec un énorme tas d'argent à ses pieds » ${ }^{(18)}$. Comme le héros, envahi par la crainte, faisait mine de s'enfuir, la femme le retint en l'appelant par son nom. Elle lui promit de lui donner tout l'argent qu'il voudrait, à condition qu'il délaissât la fille du prévôt et qu'il s'unît à elle. Puis elle déclina son identité - elle avait pour nom Méridienne - et prévint les soupçons de Gerbert : qu'il se garde de voir en elle le piège d'un démon succube! Il pouvait, assura-t-elle, avoir confiance, car l'argent qu'elle lui proposait n'était pas le fruit d'une illusion. Brûlant d'échapper à la pauvreté, Gerbert se livra corps et âme à Méridienne. De retour chez lui, il paya ses dettes, « s'acheta du mobilier, embaucha des serviteurs, accumula des parures et de l'argent, se fortifia en mangeant et en buvant ${ }^{(19)}$. Méridienne, grâce à sa connaissance du passé, fit accéder Gerbert aux sommets de la gloire; moyennant un nouvel hommage rendu à sa maîtresse, le héros progressa si bien dans la hiérarchie ecclésiastique qu'il finit par devenir pape. Cependant, lorsqu'il célébrait la messe, il s'abstenait de goûter au sang et au corps du Christ, « soit par crainte, soit par respect » ${ }^{(20)}$. Enfin, un jour qu'il officiait dans une église nommée Jérusalem, il comprit que sa mort approchait et, voyant Méridienne s'apprêter à le recevoir auprès d'elle, confessa la totalité de ses fautes devant le peuple et le clergé. Il mena le restant de ses jours une vie de pénitent, avant de mourir en odeur de sainteté.

Comme 1'a montré Laurence Harf-Lancner ${ }^{(21)}$, la trame narrative de ce récit comporte d'indéniables similitudes avec celle de Lanval : dans les deux cas, une femme surnaturelle procure au héros l'aisance matérielle et les honneurs sociaux, en échange d'une soumission qui emprunte sa forme à un lien vassalique. Mais la femme, au nom signifiant de Méridienne, est devenue un démon de midi, et semble empêcher le héros d'accéder aux sacrements chrétiens; sans que le texte se prononce explicitement à ce sujet, l'alliance que Gerbert a nouée avec sa compagne est exclusive d'une autre alliance, celle qui unit l'homme à Dieu. Cette relecture négative du pacte correspond à une réaction cléricale au modèle courtois des récits féeriques, dont Anita Guerreau-Jalabert a dégagé la portée socio-historique : face aux récits courtois qui projettent dans l'imaginaire une idéalisation de la fine amor, les clercs répondent par des récits diabolisant la femme et l'amour qu'elle suscite ${ }^{(22)}$.

L'examen du thème du don corrobore cette analyse. Dans Lanval, les dons de la fée font l'objet d'une valorisation entière et univoque. Le pacte entre le héros et la femme surnaturelle est validé par la structure même du récit, puisque les deux protagonistes finissent par partir ensemble, heureux, en Avalon. Dans l'histoire de Gerbert, à l'inverse, les biens mondains sont soumis à un jugement clairement défavorable. Le héros, dès l'abord, se révèle incapable de tirer un quelconque profit spirituel de sa pauvreté : son dénuement, loin de le ramener à l'étude et à la sagesse, le plonge dans une forme d'acédie proche de la désespérance, et éveille en lui une cupidité inextinguible. Méridienne ne

(17) Trad. cit., p. 258. Texte latin : ...substancia denique penitus direpta, domi solus residet, sui negligens, hirtus et squalidus, horridus et incultus (éd. cit., p. 352).

(18) Trad. cit., p. 259. Texte latin :...feminam ibi reperit inaudite pulcritudinis, maximo insidentem panno serico, habentem coram positum maximum denariorum aceruum (éd. cit., p. 352).

(19) Trad. cit., p. 261. Texte latin : ...supellectile ditatur, familia crescit, uestium mutatoriis et ere cumulatur, cibariis et potu stabilitur (éd. cit., p. 356).

(20) Trad. cit., p. 263. Texte latin : ...ob timorem uel ob reuerenciam (éd. cit., p. 360).

(21) Laurence Harf-Lancner, Les Fées au Moyen Âge. Morgane et Mélusine. La naissance des fées, Paris, Champion, 1984, pp. 133-138 et 395-396 sur 1'histoire de Gerbert.

(22) Anita Guerreau-Jalabert, «Des fées et des diables. Observations sur le sens des récits mélusiniens au Moyen Âge ", Mélusines continentales et insulaires, éd. Jeanne-Marie Boivin et Proinsias Mac'Cana Paris, Champion, 1999, pp. 105-137. 
s'y trompe pas : en faisant miroiter à Gerbert un immense tas d'argent, elle flatte en lui un vice déjà bien affirmé. Ici comme dans les représentations des enfers étudiées par Jérôme Baschet, "l'avarice, désir de possession matérielle, est en image strictement associée à l'or et aux formes monétaires » ${ }^{(23)}$. Par la suite, les bienfaits de la dame profitent avant tout au héros. Ils assouvissent chez lui une triple soif de richesse, de savoir et d'honneur, qui recouvre l'ensemble des désirs associés depuis saint Augustin au vice d'avaritia ${ }^{(24)}$. Certes, comme pour contrebalancer ce portrait peu flatteur, le texte précise que Gerbert « devint le pain des affamés et le vêtement des pauvres, le prompt sauveur de tous les opprimés » ${ }^{(25)}$ : sans être première, l'idée d'une redistribution des dons, amplement développée dans Lanval, apparaît fugitivement. Elle ne remet pas en cause, toutefois, la hiérarchie posée par le récit : l'accaparement personnel des biens mondains, représentés sous les espèces de l'argent et de la vaine gloire, y est marqué au sceau de l'avarice.

La critique chrétienne de l'avarice se retrouve dans le Miracle de Théophile de Rutebeuf. Selon le canevas original de la légende, qui remonte au VI siècle, le clerc Théophile, à un moment de désarroi, conclut un pacte avec le diable. Il en est quelque temps plus tard délivré par la Vierge. Ce scénario n'implique pas nécessairement que soit abordée la question du don : racontant la légende dans un sermon sur la nativité de la Vierge, Fulbert de Chartres, au début du XI ${ }^{\mathrm{e}}$ siècle, ne fait aucune place à cette thématique $^{(26)}$.

L'importance qu'elle revêt dans le texte de Rutebeuf n'en est que plus frappante. Le Miracle s'ouvre sur un monologue par lequel Théophile se plaint d'avoir perdu tous ses biens à force de dons : «Tout ai doné et despendu » (v. 3) ${ }^{(27)}$. On devine aussitôt que l'évêque a sa part de responsabilité dans cet état de pauvreté (v. 6-8). De fait, le prélat a retiré sa charge à Théophile alors qu'il était en dette vis-à-vis du héros, car celui-ci lui avait cédé l'évêché (v. 288-383). L'évêque occupe donc structurellement le même rôle que le roi de Lanval, celui d'un supérieur ayant privé un inférieur de ses subsides ; mais il ajoute à cette faute une ingratitude coupable vis-à-vis de l'abnégation et du renoncement du clerc. Sur le plan rhétorique, les bonnes et les mauvaises fortunes du héros sont soulignées par un indice qui contribue à rapprocher le Miracle et Lanval: dans les deux cas, l'adverbe initial " or » indique qu'un changement est advenu dans la situation du protagoniste, autrefois en meilleure posture (Lanval v. 33, Miracle v. 33, 36, 37, 42, 43 ) ; dans les deux cas, un même « or » indiquera le second revirement du destin héroïque, une fois conclu le pacte avec l'être surnaturel (Lanval v. 140, 154 ; Miracle v. 347 349, 368, 373...). Et dans le Miracle comme dans Lanval, ce pacte est pourvu d'une

(23) Jérôme Baschet, "Les sept péchés capitaux et leurs châtiments dans l'iconographie médiévale », dans Carla Casagrande et Silvana Vecchio, Histoire des péchés capitaux au Moyen Âge, Paris, Aubier, 2009 [éd. or. italienne : 2000, trad. fr. : 2003], pp. 339-385 : p. 376.

(24) Augustin nomme avarice générale le désir d'argent, de savoir et de pouvoir; il la distingue de l'avarice particulière, qui ne vise que l'argent. Si les siècles ultérieurs retiennent surtout la seconde, la première reparaît ici et là chez les théologiens. Voir Carla Casagrande et Silvana Vecchio, Histoire des péchés capitaux..., op. cit., pp. 158-163.

(25) Trad. cit., p. 261. Texte latin :...fit panis esuriencium, uestis egencium, et omnis oppressionis prompta redempcio (éd. cit., p. 356).

(26) Fulbert de Chartres, Sermones ad populum, Sermo IV De nativitate beatissimae Mariae virginis, PL 141, col. 323 c-d. Voici comment se noue le pacte avec le diable (la traduction est mienne) : «Ce Théophile donc, s'étant installé dans le pays d'un évêque des Ciliciens, comme un écrit l'atteste, tomba dans la désespérance à cause de ses infortunes, tant et si bien qu'il se tourna vers un magicien juif, requérant son aide et son conseil. Après avoir par le truchement de celui-ci parlé avec le diable, il renia la foi chrétienne, adora le diable et lui remit une lettre manuscrite faisant état de sa soumission, à laquelle il apposa le sceau de son anneau. »Comme on le voit, le texte ne précise pas que Théophile ait agi par cupidité.

(27) «J'ai tout donné et dépensé » (trad. cit., p. 39). 
nette valeur compensatoire : l'arrivée providentielle de Salatin, émissaire du diable, répare l'attitude de l'évêque, pour apporter à Théophile la richesse et la gloire.

Les scènes d'ouverture du lai et du Miracle traitent ainsi de façon similaire les thèmes du don et du dépouillement. La suite du Miracle interdit de pousser plus loin l'analogie, et conduit au contraire à remarquer combien les deux textes divergent. L'usage des dons d'origine surnaturelle établit en effet un rapport d'opposition entre les protagonistes. Là où Lanval et Gerbert redistribuent, fût-ce, pour le second, avec parcimonie et pour ainsi dire du bout des lèvres, les dons qu'ils ont reçus, Théophile, devenu la créature du diable, bafoue toutes les vertus chrétiennes : selon le contre-credo que lui prescrit son nouveau maître aux vers 256-284, il doit cultiver l'orgueil et la fourberie, se garder de soulager les malades et de donner aux miséreux. Par la suite, il adopte une attitude insultante et blasphématoire, incarnant, outre l'avarice, les péchés capitaux d'orgueil et de colère (v. 299, 319, 329, 336...). Sa repentance et sa conversion s'imposent par conséquent avec une nécessité criante.

Si l'on fait retour à présent sur la vie de Gerbert, il apparaît qu' elle se situe, du point de vue de sa genèse, à la croisée de la légende de Théophile et du lai de Lanval ou, plus justement, à la croisée des patrons narratifs actualisés dans ces récits. À l'époque de Gautier Map, les clercs connaissent et travaillent depuis longtemps le thème du pacte avec le diable, représenté par exemple dans l'histoire de Théophile. À ce thème se superpose l'introduction des fées par les textes vernaculaires, et l'arsenal de motifs associés : isolement du héros dans la nature sauvage, éventuellement près d'un plan ou d'un cours d'eau, beauté extraordinaire de la dame, etc. Gautier Map, et les autres clercs qui réélaborent à la même période les récits féeriques, calquent sur le schéma fourni par les textes vernaculaires le thème plus ancien du pacte démoniaque, qui leur permet de diaboliser à peu de frais la femme surnaturelle.

Dans les textes examinés jusqu'ici, le dispositif d'ensemble obéit à quelques traits constants. Le héros, clerc ou chevalier, ruiné de son propre fait (Gerbert) ou à cause d'un supérieur censé être le "père des pauvres » ${ }^{(28)}$ (Lanval, Théophile), rencontre un être surnaturel qui, en échange d'un serment d'allégeance, le rétablit dans une situation de prospérité inédite. La théorie de Marcel Mauss invite à comprendre comme un contre-don le rapport de soumission entre le héros et ses donateurs, et comme un scandale ou comme un manquement l'interruption de la circulation des biens par les détenteurs du pouvoir. Néanmoins, si cette théorie éclaire parfaitement la logique narrative à l'œuvre dans Lanval, elle ne suffit pas à rendre compte des deux autres textes. Pour aller plus loin, il faut procéder, suivant la recommandation d'Eliana Magnani, à une « historicisation des concepts ", " préalable indispensable à un usage raisonné des théories en sciences sociales " ${ }^{(29)}$. En l'occurrence, la question du don se pose au Moyen Âge dans le cadre d'une pensée chrétienne, dont les conceptions touchent autant à la notion de $\mathrm{ca}^{-}$ ritas qu'à la valeur des biens matériels et terrestres : accaparés par un individu, ceux-ci mettent en péril son salut, en suscitant en lui le péché d'avarice. Ces conceptions expliquent la nature de l'être surnaturel qui, chez Gautier Map et Rutebeuf, pourvoit d'honneurs et de richesses le héros démuni : il s'agit dans les deux cas d'un être diabolique.

(28) Sur cette expression appliquée à l'évêque et au roi, voir Michel Mollat, Les Pauvres au Moyen Âge, Bruxelles, Complexe, 2006 [première éd. : 1978], pp. 53-58, et Priscille Aladjidi, Le roi père des pauvres, op. cit.

(29) Eliana Magnani Soares-Christen, «Les médiévistes et le don. Avant et après la théorie maussienne », Don et sciences sociales. Théories et pratiques croisées, dir. Eliana Magnani, Dijon, Éditions Universitaires de Dijon, 2007, pp. 15-28:p. 28. 


\section{Changer de paradigme : le jongleur et le Christ}

Le dernier dossier retenu date du XIII ${ }^{\mathrm{e}}$ siècle. Il rassemble les versions latine et française d'un miracle opéré au bénéfice d'un jongleur par le Volto Santo de Lucques, effigie supposée avoir été sculptée par Nicodème ${ }^{(30)}$. Comme on le verra, la version française propose une configuration originale autour de la question des biens mondains, de leur prix et de leur circulation.

La version latine, dans les manuscrits, a été tantôt copiée séparément, tantôt intégrée à une liste de treize miracles attribués à l'image sacrée ${ }^{(31)}$. Un jeune homme gaulois, parti pour Jérusalem en pèlerinage, s'arrêta à Lucques pour contempler la statue du Seigneur. Parvenu auprès de l'effigie, il fut inondé par d'abondantes larmes. Voyant que la troupe des fidèles « s'avançait humblement, chargée de présents, jusqu'au très saint pied du Voult, lui, qui était pauvre de bien mais riche de mérites, tout honteux et s'estimant indigne, en homme qui s'avançait devant les autres les mains vides, commença à se demander ce qu'il devait faire » ${ }^{(32)}$. Attrapant son instrument de musique, et « souhaitant offrir, en guise de présent, le don qu'il pouvait apporter, à savoir des louanges accompagnées de sa mélodie, il commença à chanter des hymnes en l'honneur de la très sainte croix, si doucement qu'il charma par son exquise suavité l'ouïe de tous les assistants » ${ }^{(33)}$. Pris de compassion, Dieu décida de lui témoigner sa grâce par un don : «aussitôt, chose merveilleuse à dire, le soulier d'argent qui chaussait le pied droit du saint Voult, par la seule puissance de Dieu, bondit au loin et tomba dans le sein du chanteur $"{ }^{(34)}$. Tout à la fois émerveillé et embarrassé par ce don insolite, le jeune homme - qui possède tous les attributs d'un jongleur, bien que le texte n'emploie jamais les termes de joculator ni d' histrio - se retira un moment dans l'église pour réfléchir. Finalement, « il revint dans la chapelle, s'avança, suppliant et plein d'humilité, vers le pied du Voult très glorieux, ce qu'il redoutait auparavant quand il avait les mains vides, et donna en présent au Dieu tout-puissant, en l'honneur de la sainte croix, ce même soulier d'argent qui lui avait été offert en ce lieu par la grâce divine ${ }^{(35)}$. La cité entière accourut et cria au miracle. Le récit se clôt sur une notation étiologique : depuis ce jour-là, le soulier n'a plus jamais pu s'adapter exactement au pied droit du crucifix.

La version française est rédigée en laisses décasyllabiques, dans la forme canonique de la chanson de geste. Le miracle du Volto Santo y occupe une laisse longue de plus de cent vers (v. 403-509), au sein d'un récit servant de prologue à la Venjance Nostre Seigneur dans l'unique manuscrit où il est conservé (Turin, BN, L.II.14). Après avoir raconté la translation du crucifix jusqu'à Lucques, le narrateur annonce le récit d'une

(30) Je laisse de côté la traduction du récit latin composée en prose française par le carme Jean Golein vers 1401-1402 - donc bien au-delà du cadre chronologique de cet article. Cette traduction fait partie des Festes nouvelles de Jean Golein, série de récits ajoutée à la traduction de la Légende dorée par Jean de Vignay.

(31) Voir Jean-Claude Schmitt, "Cendrillon crucifiée. À propos du Volto Santo de Lucques (XIII'$\mathrm{XV}^{\mathrm{e}}$ siècle) ", Le Corps des images. Essais sur la culture visuelle au Moyen Âge, Paris, Gallimard, 2002, pp. 217-271: pp. 259-271.

(32) ...ad sanctissimum vultus pedem humillime cum suis accedere muneribus, pauper rebus sed meritis dives, quasi qui ante alios vacua manu accederet, se existimans indignum verecundus, quid ageret, dubitare cepit. Ici et dans la suite du texte, les traductions sont miennes.

(33) ...quod poterat donum sue scilicet modulationis laudes vice muneris offerre cupiens, ad honorem sanctissime crucis hymnos tanta dulcedine cantare cepit ut omnium astantium nimia delectatione demulceret auditum.

(34) ...Illico enim, quod est mirabiliter dictum, argenteum calcamentum de destro sancti vultus pede sola Dei potentia procul exiliens in gremium cecidit cantantis.

(35) ... regressus est in capellam et ad gloriosissimi vultus pedem, quod prius verebatur inops, suppliciter et cum omni humilitate accedens argenteum illud calciamentum divina sibi gratia largitum omnipotenti Deo ibidem ad honorem sancte crucis munus obtulit. 
«merveille» (v. 402). Le dénommé Genois, jongleur de son état, avait pour coutume de ne pas rompre le jeûne « desqu'il l'eüst gaaigniet au chanter » (v. 414) ${ }^{(36)}$. Parvenu à Lucques un dimanche, il s'installa et chanta tout l'après-midi. Les sept cents hommes qui s'assemblèrent pour l'écouter «ne li dounerent un denier mounaé » (v. 420) ${ }^{(37)}$. En fin de journée, Genois entra dans l'église, aperçut le Voult récemment arrivé, s'enquit de son identité et, pour l'amour du Christ, se mit à chanter en s'accompagnant de sa vielle. L'effigie, animée par le saint Esprit, envoya au jongleur son soulier d'or et d'argent, tout incrusté de pierres. Sur ce, Genois sortit pour déjeuner. En le voyant muni du précieux soulier, les habitants de la cité alertèrent l'évêque, qui enjoignit au jongleur de rendre son bien à l'image sacrée : si le soulier, dit-il, était une seconde fois lancé par le crucifix au jongleur, celui-ci pourrait le garder sans contredit. On ramena le soulier au saint Voult qui, « de grant ire embrasés » (v. 455), le renvoya aussitôt à la face de l'assistance et demanda à ce qu'il soit « chierement comparé » au jongleur (v. 459) ${ }^{(38)}$. L'évêque pria donc Genois de vendre le soulier à la cité. Le jongleur répondit qu'il n'en demanderait pas plus qu'on ne voudrait lui en donner. Après avoir réuni la somme de deux cents livres, on rapporta le soulier à l'effigie ; mais l'objet n'était " point rachatés » ${ }^{(39)}$. On remplit alors le soulier d'or et d'argent. Cette fois, quand Genois ramena le soulier au saint Voult, celui-ci l'enfila et retrouva son inertie habituelle. Genois, « de son avoir que Dex li ot presté » (v. 480) ${ }^{(40)}$, fit apprêter un repas pour les pauvres de Lucques, puis donna à Dieu l'argent restant. Une fois reparti, il rencontra des hérétiques qui lui infligèrent le martyre et périt sanctifié.

Martine Clouzot a replacé ces deux récits dans un groupe d'exempla érigeant le jongleur en modèle ${ }^{(41)}$. Ces exempla peuvent être considérés comme des illustrations narratives, ou encore des prolongements pastoraux, du paradoxe développé par Bernard de Clairvaux : dans une lettre adressée à Ogier du mont Saint-Éloi, le docteur explique que le «jongleur de Dieu » est le sujetd'une humiliation qui vaut, dans la pensée chrétienne, élection. Le jongleur n'est réhabilité que sur le plan métaphorique, et non pour son status social ${ }^{(42)}$. De la même manière, dans les récits de miracle, «le jongleur n'est pas traité pour lui-même, mais pour les exemples édifiants qu'il incarne : la pauvreté, la conversion, la charité ${ }^{(43)}$. Encore faut-il comprendre comment les histoires du Volto Santo articulent à ces valeurs chrétiennes la question des échanges et du don.

Dans le récit latin, le Volto Santo, par son formidable lancer de soulier, rétribue le jeune musicien ; celui-ci, désireux de préserver le caractère gratuit de son chant de

(36) « avant d'avoir gagné [son déjeuner] par son chant».

(37) « ne lui donnèrent pas un seul denier de monnaie».

(38) « enflammé d'une grande colère »... « acheté au prix fort».

(39) «pas racheté ». Les vers 468-469 donnent : "Au saint vous a reporté son soler. / Et dist Genois : "Il n'est point rachatés". » Comme le signale l'éditeur dans l'apparat critique de la p. 44, le jeu des pronoms et des sujets, dans ces vers et les précédents, est assez ambigu. Dans le vers 468, le jongleur paraît porter lui-même le soulier au crucifix. Dans le vers 469 , le sujet semble être également Genois, si l'on se fie aux autres vers construits sur ce schéma ( $c f$. les v. 460, 462, 465). Or il n'est pas logique que le jongleur se prononce lui-même sur le prix du soulier. En effet, outre qu'il n'a d'abord réclamé du soulier que ce qu'on voudrait bien lui en donner, il finit par se dépouiller de tous ses biens. En revanche, il revient au Voult d'exiger le rachat du soulier, puis, dans la scène suivante, d'accepter de reprendre son soulier désormais renchéri : le Voult paraît seul à même de décider si le prix payé par la cité est suffisant. Comment comprendre alors le vers 469 ? On peut imaginer que Genois se fait le messager des volontés du Voult, qu'il retransmet au discours direct aux habitants de la cité.

(40) « avec le bien que Dieu lui avait prêté ».

(41) Martine Clouzot, «Un intermédiaire culturel au XIII ${ }^{\mathrm{e}}$ siècle : le jongleur », Bulletin du centre d'études médiévales d'Auxerre [En ligne], Hors série $\mathrm{n}^{\circ} 2$, mis en ligne le 24 janvier 2009, URL : http://cem.revues.org.

(42) Outre l'article de Martine Clouzot, on peut lire à ce sujet Michel Zink, Poésie et conversion au Moyen Âge, Paris, Presses Universitaires de France, 2003, pp. 164-176.

(43) Martine Clouzot, «Un intermédiaire culturel... », art. cit., § 23. 
louanges, rend son bien à l'image sacrée. Si le soulier ne colle plus ensuite au pied de l'effigie, c'est peut-être parce que le Christ n'accepte qu'à demi ce qu'il avait, après tout, octroyé en paiement au jongleur. La position singulière du soulier pourrait traduire l'ambiguïté du statut accordé à l'objet, tour à tour paiement du chant et don au crucifix. Le miracle mettrait à l'épreuve le désintéressement du musicien tout en lui fournissant le moyen d'acquitter son offrande. Que l'on admette ou non cette hypothèse, le trajet du soulier équivaut à un aller-retour dirigé, en dernier ressort, vers le Ciel. Il ne concerne guère la communauté des hommes, qui n'intervient que pour admirer, louer et attester le miracle divin.

Le récit français, à l'inverse, confère une large place à la représentation de la cité terrestre. Il met au premier plan, autant que l'effigie, le jongleur; le fait que celui-ci acquière un nom dans la translation linguistique marque son accès au statut de protagoniste. Aux côtés du jongleur et du Voult, le texte présente plusieurs acteurs sociaux : l'évêque, les habitants de la cité et, au sein de ceux-ci, le groupe plus restreint des pauvres. Les échanges entre ces différents acteurs peuvent être interprétés à la lumière des dons pro anima, par lesquels les riches laïcs assuraient au Moyen Âge leur salut ${ }^{(44)}$. Ces dons engageaient une multiplicité de partenaires : les donateurs, les communautés ecclésiastiques, les défunts et les pauvres, auxquels s'ajoutait le saint, la vierge ou, ici, le Christ au centre du culte local. Ils impliquaient ainsi bien plus de deux parties, ce qui rend délicate l'application de la théorie de Marcel Mauss : comme l'écrit Michel Lauwers, « la pratique du don ne se limitait donc pas à un simple échange entre les nobles et l'Église, pas plus qu'elle ne se réduisait au jeu du don (matériel) et du contre-don (spirituel) évoqué dans le discours ecclésiastique. Car seule l'Église pouvait assurer la circulation des dons et la transformation des biens matériels en biens spirituels ${ }^{(45)}$. De fait l'Église, faisant office de «pivot», transformait en aumônes les donations laïques ; grâce à sa fonction médiatrice, elle exerçait un contrôle symbolique et concret sur la circulation des biens.

En démarquant ce type d'échanges, on pourrait imaginer que Genois fasse un don, éventuellement augmenté par un miracle du Voult, puis que ce don soit redistribué aux pauvres par l'évêque. Or le récit français donne à voir tout autre chose. En premier lieu, l'évêque, "père des pauvres ", déroge à son rôle idéal, d'une manière tout aussi nette que dans le Miracle de Théophile. Le prélat a le front de contester le don du saint Voult au jongleur. En refusant que le soulier puisse revenir à un autre, il paraît en revendiquer la propriété, pour lui ou pour l'église - alors que l'objet, très visiblement, appartient avant tout à l'image sacrée ${ }^{(46)}$. Aussi le Voult, pour réaffirmer ses droits sur son soulier, en est-il réduit à manifester sa colère à l'assistance. La seconde distorsion, qui découle de la première, réside dans la fonction concédée au jongleur. Genois joue un rôle comparable à celui de Lanval dans le lai de Marie de France : grâce à l'intervention surnaturelle, il se substitue à l'évêque dans l'accomplissement de son devoir d'aumône ; assu-

(44) Voir Dominique Iogna-Prat, Ordonner et exclure. Cluny et la société chrétienne face à l'hérésie, au judaïsme et à l'islam (1000-1150), Paris, Aubier, 1998, pp. 186-252; Michel Lauwers, La mémoire des ancêtres, le souci des morts. Morts, rites et société au Moyen Âge, Paris, Beauchesne, 1997 ; Eliana Magnani, «Le don au Moyen Âge : pratique sociale et représentations. Perspectives de recherche », Revue du M.A.U.S.S. 19, 2002, pp. 309-322.

(45) Michel Lauwers, La mémoire des ancêtres..., op. cit., p. 192.

(46) Le fait qu'une image soit considérée comme la propriétaire de ses habits ou de ses ornements est largement attesté, à partir du XIV e siècle au moins, dans le culte rendu aux images de la Vierge. On trouve ainsi des inventaires, des testaments et des livres de comptes précisant que c'est à telle ou telle statue, et non à l'église, qu'appartiennent des habits ou des bijoux donnés par les fidèles. Voir les témoignages relevés dans le catalogue d'exposition d'Antonio Cea Gutiérrez, Religiosidad popular. Imágenes vestideras, Zamora, Caja España, 1992, notamment pp. 39-42, et les exemples donnés dans Madonna della Laguna. Simulacri “da vestire” dei secoli XIV-XIX, éd. Riccarda Pagnozzato, Roma, Regione del Veneto, Fondazione Giorgio Cini ed Istituto della Enciclopedia italiana, 1993. 
mant le rôle de l'Église, il redistribue lui-même l'argent qu'il a gagné. Ce dispositif subvertit les pratiques contemporaines : la circulation des biens est l'effet d'une relation directe entre le Christ et le jongleur, qui nie ou oblitère la médiation ecclésiasti que $^{(47)}$.

Si le récit estompe la fonction médiatrice de l'église, il véhicule en revanche une conception toute cléricale des pauvres et de la pauvreté. Pour citer Jean-Claude Schmitt, les pauvres, dans le récit français, «incarnent [...] la communauté du "peuple" de Dieu », et apparaissent comme «les intermédiaires privilégiés entre l'Église et la divinité » ${ }^{(48)}$. Le rachat du soulier, en effet, est justifié par l'affectation allouée au montant obtenu : le texte laisse entendre que le Christ a «presté » de l'argent au jongleur à la condition expresse que la somme soit reversée aux «povres gens [...] de la cité» (v. 480-481). Quant à Genois, il entretient avec les pauvres un double rapport d'analogie et d'opposition. L'analogie ressort d'un commun dépouillement matériel, illustré dans le thème du jeûne forcé ou consenti : parvenu à Lucques avec l'espoir de trouver à déjeuner, Genois choisit précisément de conférer à l'aumône finale la forme d'un repas. L'opposition, de son côté, se comprend dans le cadre des représentations propres au Moyen Âge chrétien. Les clercs et les théologiens répètent à l'envi que les dons aux jongleurs privent les pauvres de leur dû ${ }^{(49)}$. Comme pour ne pas usurper les biens qui lui sont accordés, Genois s'en sert pour régaler les pauvres de la ville ; il est, d'une certaine manière, un mauvais pauvre qui se convertit et se rachète en donnant aux bons pauvres. Ceux-ci permettent que s'opère la commutatio du bénéfice pécuniaire en aumône et, pour Genois, de l'aumône en salut spirituel : le jongleur renonce pour finir aux biens terrestres en faveur des biens célestes, incommensurables aux premiers. Les vers 485-486 sonnent comme une justification théologique de son dépouillement volontaire : «Pour nul avoir ne voet estre encombrés, / ne ne voet estre des chieus desiretés " ${ }^{(50)}$. Plus loin, le martyre du héros montre qu'il y a place pour tous au royaume des cieux : par son aumône comme par son trépas, le jongleur fait figure d'élu, ici-bas et dans l'au-delà.

Il reste un dernier point, qui intéresse directement la pensée économique à l'œuvre dans le récit. Tandis que la version latine ne prête guère d'attention aux circonstances matérielles du miracle, le jongleur est aux prises dans la version française avec un univers éminemment concret. Son nom, Genois, constitue un premier indice. Gênes est au Moyen Âge une grande puissance bancaire et commerciale ${ }^{(51)}$. Genois pourrait conjoindre en lui, virtuellement, le ménestrel et le marchand - deux métiers "illicites » dans leurs rapports au don et à l'argent ${ }^{(52)}$. Et effectivement, les préoccupations du personnage sont au départ fort peu spirituelles : s'il chante, c'est d'abord non pas pour prier, mais pour gagner son déjeuner; il espère le gagner en espèces sonnantes et trébuchantes, comme en témoigne la mention du «denier mounaé » (v. 420). Ce matérialisme se confirme lors du rachat du soulier, dont le prix se mesure en livres et en poids de métal. Il correspond à une évolution en vertu de laquelle les échanges tendent à se monéta-

(47) Pour une autre interprétation quant aux rôles respectifs de l'évêque et du jongleur, voir Jean-Claude Schmitt, «Cendrillon crucifiée... », art. cit., pp. 237-252.

(48) Ibid., p. 242.

(49) Martine Clouzot, «Un intermédiaire culturel... », art. cit., § 37 sq.

(50) Le jongleur «ne veut être entravé par aucun bien, car il ne veut pas perdre cet héritage qu' est le ciel». J'adopte pour le vers 486 le découpage proposé par l'éditeur dans l'apparat critique de la p. 51.

(51) Je remercie Patrick Henriet de m'avoir donné cette indication.

(52) L'expression est celle de Jacques Le Goff, « Métiers licites et métiers illicites dans l'Occident médiéval », Pour un autre Moyen Âge, Paris, Gallimard, 1977, pp. 91-107. L'idée d'une condamnation chrétienne du marchand est à nuancer toutefois, la question évoluant au cours du XIII ${ }^{\mathrm{e}}$ siècle : voir André Vauchez, «Homo mercator vix aut numquam potest Deo placere : quelques réflexions sur 1'attitude des milieux ecclésiastiques face aux nouvelles formes de l'activité économique au XII et au début du XIII ${ }^{\mathrm{e}}$ siècle ", Actes des congrès de la Société des historiens médiévistes de l'enseignement supérieur public. Le marchand au Moyen Age, 19e congrès, Reims, 1988, pp. 211-217. 
riser au cours du XIII ${ }^{\mathrm{e}}$ siècle, autant dans le domaine des transactions commerciales que dans celui des donations pieuses ${ }^{(53)}$. Cette évolution, toutefois, ne résorbe pas complètement l'étrangeté de la scène du rachat, au cours de laquelle le Christ, par le biais de son effigie, se livre à un véritable marchandage. En faisant endosser au saint Voult le rôle d'un marchand de tapis, le texte brouille la frontière entre la vente et l'aumône, le don et l'échange commercial; il superpose deux types de transactions habituellement distincts ${ }^{(54)}$. Mais par un tour de passe-passe qui s'assimile à une ruse du ciel, la vente occasionne le don. Cette opération permet au Christ de tester et de réaffirmer la relation de patronage qu'il entretient avec la ville de Lucques, en mettant à contribution la générosité de ses fidèles. Elle lui permet aussi de contraindre l'évêque à s'acquitter de son devoir d'aumône. Grâce à la médiation de l'effigie, Genois extorque de l'argent à l'évêque et à sa cité, comme peut le faire le «magicien voleur » vis-à-vis de Charlemagne dans les textes étudiés par Philippe Haugeard ${ }^{(55)}$.

Le parcours proposé m'a amenée à utiliser diversement la théorie de Marcel Mauss. Si le lai de Lanval se prête à une application assez immédiate de cette théorie, les histoires des clercs Gerbert et Théophile appellent des nuances, qui reviennent à intégrer à l'analyse la suspicion chrétienne à l'encontre de l'enrichissement individuel. Quant au miracle du Volto Santo, il a nécessité, d'une manière plus radicale, de changer de paradigme interprétatif, pour inscrire le récit dans les modalités médiévales et chrétiennes des échanges entre ciel et terre. À travers différents mésusages du don, ces textes ordonnent, dans la sphère de l'imaginaire, plusieurs fonctions sociales. Sur le plan structurel, chacun met en tension deux couples de pôles. D'une part, face à un dominant - roi ou évêque - ayant manqué à ses devoirs de largesse, de charité ou de rétribution, se positionne un héros démuni - chevalier, clerc ou jongleur. Ce dernier binôme illustre les rapports d'homologie et de rivalité entre les deux figures ${ }^{(56)}$ : Théophile et Genois, visà-vis de l'évêque, occupent des places similaires. Leur proximité fournit un argument supplémentaire aux analyses faisant de Théophile un double du jongleur Rutebeuf. D'autre part, face aux carences des biens et des acteurs terrestres, chaque texte met en scène un être surnaturel investi d'une fonction compensatoire, qui relève tour à tour, selon que les textes promeuvent des valeurs cléricales ou aristocratiques, du merveilleux, du diabolique ou du divin.

(53) Voir Priscille Aladjidi,Le roi père des pauvres, op. cit., pp. 131-180, et Michel Lauwers, La mémoire des ancêtres..., op. cit., pp. 474-479.

(54) Sur les distinctions qu'opèrent les textes médiévaux entre ces deux types de transactions, voir Michel Lauwers, ibid., pp. 202-203.

(55) Philippe Haugeard, «Le magicien voleur et le roi marchand : essai sur le don dans Renaut de Montauban», Romania 123, 2005, pp. 292-320.

(56) Sur ce face-à-face, voir Carla Casagrande et Silvana Vecchio, «Clercs et jongleurs dans la société médiévale », Annales ESC 5, 1979, pp. 913-928. 